Pacific Journal of Mathematic 


\section{COMMUTING SPECTRAL MEASURES ON HILBERT SPACE}

JOHN WERMER

1. Introduction. By a "spectral measure" on Hilbert space $H$ we mean a family of bounded operators $E(\sigma)$ on $H$ defined for all Borel sets $\sigma$ in the plane. We suppose:

(i) If $\sigma_{0}$ denotes the empty set and $\sigma_{1}$ the whole plane, then

$$
E\left(\sigma_{0}\right)=0, E\left(\sigma_{1}\right)=I
$$

where $I$ is the identity.

(ii) For all $\sigma_{1}, \sigma_{2}$,

$$
E\left(\sigma_{1} \cap \sigma_{2}\right)=E\left(\sigma_{1}\right) E\left(\sigma_{2}\right)
$$

and for disjoint $\sigma_{1}, \sigma_{2}$,

$$
E\left(\sigma_{1} \cup \sigma_{2}\right)=E\left(\sigma_{1}\right)+E\left(\sigma_{2}\right)
$$

(iii) There exists a constant $M$ with $\|E(\sigma)\| \leq M$, all $\sigma$. It follows that $E(\sigma)^{2}=E(\sigma)$ for each $\sigma$, and $E\left(\sigma_{1}\right) E\left(\sigma_{2}\right)=0$ if $\sigma_{1}, \sigma_{2}$ are disjoint.

Mackey has shown in [3], as part of the proof of Theorem 55 of [3], that if $E(\sigma)$ is a spectral measure with the properties just stated, then there exists a bicontinuous operator $A$ such that $A^{-1} E(\sigma) A$ is self-adjoint for every $\sigma$. In a special case this result was proved by Lorch in [2]. We shall prove:

THEOREM 1. Let $E(\sigma)$ and $F(\eta)$ be two commuting spectral measures on $H$; that is,

$$
E(\sigma) F(\eta)=F(\eta) E(\sigma)
$$

for every $\sigma, \eta$. Then there exists a bicontinuous operator $A$ such that $A^{-1} E(\sigma) A$ and $A^{-1} F(\eta) A$ are self-adjoint for every $\sigma, \eta$.

As a corollary of Theorem 1, we shall obtain:

Received March 4, 1953.

Pacific J. Math. 4 (1954), 355-361 
THEOREM 2. If $T_{1}, T_{2}$ are spectral operators on $H$, in the sense of Dunford [1], and $T_{1} T_{2}=T_{2} T_{1}$, then $T_{1}+T_{2}$ and $T_{1} T_{2}$ are again spectral operators.

2. Lemmas. We shall use two lemmas in proving Theorem 1 .

LEMMA 1. Let $P_{1}, P_{2}, \ldots, P_{n}$ be operators on Hilbert space with

$$
P_{i} P_{j}=0 \quad(i \neq j), \quad P_{i}^{2}=P_{i}, \quad \sum_{i=1}^{n} P_{i}=I .
$$

Suppose that, for every set $\delta_{1}, \delta_{2}, \cdots, \delta_{n}$ of zeros and ones,

$$
\left\|\sum_{i=1}^{n} \delta_{i} P_{i}\right\| \leq M
$$

Then for every $x$ we have

$$
\frac{1}{4 M^{2}}\|x\|^{2} \leq \sum_{i=1}^{n}\left\|P_{i} x\right\|^{2} \leq 4 M^{2}\|x\|^{2}
$$

This Lemma is proved in [3, p. 147]; we include the proof for completeness. Proof. We note that

$$
\sum_{i=1}^{n}\left\|P_{i} x\right\|^{2}=\frac{1}{2^{n}} \sum\left\|\epsilon_{1} P_{1} x+\cdots+\epsilon_{n} P_{n} x\right\|^{2}
$$

where the sum is taken over all possible sets $\left(\epsilon_{1}, \epsilon_{2}, \ldots, \epsilon_{n}\right)$, where $\epsilon_{i}= \pm 1$. Hence

$$
\begin{aligned}
a_{x} & =\left\|\epsilon_{1}^{\prime} P_{1} x+\cdots+\epsilon_{n}^{\prime} P_{n} x\right\|^{2} \leq \sum_{i=1}^{n}\left\|P_{i} x\right\|^{2} \\
& \leq\left\|\epsilon_{1} P_{1} x+\cdots+\epsilon_{n} P_{n} x\right\|^{2}=b_{x}
\end{aligned}
$$

for some choice of the $\epsilon_{i}^{\prime}$ and $\epsilon_{i}$. Now

$$
b_{x}=\left\|\sum_{i=1}^{n} \delta_{i}^{+} P_{i} x-\sum_{i=1}^{n} \delta_{i}^{-} P_{i} x\right\|^{2},
$$


where the $\delta_{i}^{+}$and the $\delta_{i}^{-}$are 1 or 0 .

\section{Hence}

$$
\sum_{i=1}^{n}\left\|i_{i} x\right\|^{2} \leq 4 M^{2} \cdot\|x\|^{2}
$$

Iet now $P^{+}=\sum P_{l}$, summed over those $i$ with $\epsilon_{i}^{\prime}=1$; and let $P^{-}=\sum P_{i}$, summed over those $i$ with $\epsilon_{i}^{\prime}=-1$. Then

$$
\left(P^{+}-p^{-}\right)^{2}=p^{+}+P^{-}=1 \text { and }\left\|P^{+} x-P^{-} x\right\|^{2}=a_{x} .
$$

lience

$$
\|x\|^{2}=\left\|\left(P^{+}-P^{-}\right)^{2} x\right\|^{2} \leq\left\|P^{+}-p^{-}\right\|^{2} \cdot\left\|P^{+} x-P^{-} x\right\|^{2} .
$$

Now $\left\|n^{+}\right\| \leq M$ and $\left\|f^{-}\right\| \leq \|$and so

$$
\|x\|^{2} \leq(2 H)^{2} a_{x} \leq(2 M)^{2} \sum_{i=1}^{n}\left\|P_{i} x\right\|^{2}
$$

Lemma 2. Let $E(\sigma)$ and $F(\eta)$ be commuting spectral measures on Hilbert space. Then there is a fixed $K$ such that for any set $\sigma_{1}, \sigma_{2}, \cdots, \sigma_{n}$ of disjoint Borel sets, and set $\eta_{1}, \eta_{2}, \cdots, \eta_{n}$ of arbitrary Borel sets,

$$
\left\|\sum_{i=1}^{n} \dot{L}\left(\sigma_{i}\right) F\left(\eta_{i}\right)\right\| \leq k .
$$

Proof. Fix $x$. By (iii) there exist constants $L$ and $M$, with $\|E(\sigma)\| \leq M$, $\|F(\eta)\| \leq L$ for any $\sigma, \eta$. Let $\sigma_{n+1}$ be the complement of

$$
\bigcup_{i=1}^{n} \sigma_{i} \cdot
$$

Then

$$
\left\|\sum_{i=1}^{n} E\left(\sigma_{i}\right) F\left(\eta_{i}\right) x\right\|^{2} \leq 4 M^{2} \sum_{\nu=1}^{n+1}\left\|E\left(\sigma_{\nu}\right)\left(\sum_{i=1}^{n} E\left(\sigma_{i}\right) F\left(\eta_{i}\right) x\right)\right\|^{2}=C
$$

by Lemma 1; 


$$
C=4 M^{2} \sum_{\nu=1}^{n}\left\|E\left(\sigma_{\nu}\right) F\left(\eta_{\nu}\right) x\right\|^{2}
$$

since $E\left(\sigma_{\nu}\right) E\left(\sigma_{i}\right)=E\left(\sigma_{\nu} \cap \sigma_{i}\right)$;

$$
C=4 M^{2} \sum_{\nu=1}^{n}\left\|F\left(\eta_{\nu}\right) E\left(\sigma_{\nu}\right) x\right\|^{2},
$$

by commutativity of the $E(\sigma)$ and $F(\eta)$;

$$
C \leq 4 M^{2} \cdot L^{2} \sum_{\nu=1}^{n}\left\|E\left(\sigma_{\nu}\right) x\right\|^{2}
$$

since $\left\|F\left(\eta_{\nu}\right)\right\| \leq L$

$$
C \leq\left(4 M^{2}\right)^{2} \cdot L^{2}\|x\|^{2}
$$

by Lemma 1. Hence

$$
\left\|\sum_{i=1}^{n} E\left(\sigma_{i}\right) F\left(\eta_{i}\right)\right\| \leq 4 M^{2} L
$$

In the proof of Theorem 1 we shall use the method of Mackey in [3], together with Lemmas 1 and 2.

3. Proof of Theorem 1. By a "partition" $\pi$ of the plane we mean a finite family of Borel sets $\sigma_{1}, \sigma_{2}, \cdots, \sigma_{n}$, mutually disjoint and with union equal to the whole plane. If $(x, y)$ denotes the given scalar product in $H$, and

$$
\pi_{1}=\left(\sigma_{i}\right)_{i=1}^{n} \quad \pi_{2}=\left(\eta_{j}\right)_{j=1}^{m}
$$

are two partitions, set

$$
(x, y)_{\pi_{1}, \pi_{2}}=\sum_{i=1}^{n} \sum_{j=1}^{m}\left(E\left(\sigma_{i}\right) F\left(\eta_{j}\right) x, E\left(\sigma_{i}\right) F\left(\eta_{j}\right) y\right) .
$$

It is easily verified that the quantity $(x, y)_{\pi_{1}, \pi_{2}}$ is a scalar product in $H$. Further, it follows by Lemma 2 that the operators 


$$
P_{i j}=E\left(\sigma_{i}\right) F\left(\eta_{j}\right) \quad(i=1,2, \cdots, n ; j=1,2, \cdots, m,)
$$

satisfy the hypotheses of Lemma 1.

Hence Lemma 1 yields

$$
\frac{1}{4 K^{2}}\|x\|^{2} \leq \sum_{i=1}^{n} \sum_{j=1}^{m}\left\|E\left(\sigma_{i}\right) F\left(\eta_{j}\right) x\right\|^{2} \leq 4 K^{2}\|x\|^{2},
$$

where $K$ depends only on $\sup _{\sigma}\|E(\sigma)\|$ and $\sup _{\eta}\|F(\eta)\|$. But

$$
\sum_{i=1}^{n} \sum_{j=1}^{m}\left\|E\left(\sigma_{i}\right) F\left(\eta_{j}\right) x\right\|^{2}=(x, x)_{\pi_{1}, \pi_{2}}=\|x\|_{\pi_{1}, \pi_{2}}^{2} \cdot
$$

Finally, each $E\left(\sigma_{i}\right)$ and $F\left(\eta_{j}\right) \quad(i=1,2, \cdots n ; j=1,2, \cdots, m)$ is selfadjoint in the scalar product $(x, y)_{\pi_{1}, \pi_{2}}$, as is readily verified.

For each pair of vectors $x, y \in H$, now, let $S_{x y}$ be the disk in the complex plane consisting of all $z$ with

$$
|z| \leq 4 K^{2}\|x\| \cdot\|y\|
$$

If $S$ denotes the cartesian product of the disks $S_{x y}$ over all pairs $x, y$, then $S$ is a compact topological space, by Tychonoff's theorem. Further, as we saw above,

$$
\|x\|_{\pi_{1}, \pi_{2}}^{2} \leq 4 K^{2}\|x\|^{2}
$$

Hence by Schwarz's inequality, applied to the scalar product $(x, y)_{\pi_{1}, \pi_{2}}$, we see that the number $(x, y)_{\pi_{1}, \pi_{2}}$ lies in the disk $S_{x y}$ for every pair $x, y$. Hence there is a point $p_{\pi_{1}, \pi_{2}}$ in $S$ whose $x, y$-coordinate is $(x, y)_{\pi_{1}, \pi_{2}}$.

Let us now partially order the set of points $p_{\pi_{1}, \pi_{2}}$ in $S$ by saying that

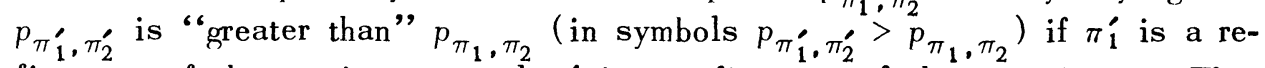
finement of the partion $\pi_{1}$, and $\pi_{2}^{\prime}$ is a refinement of the partition $\pi_{2}$. This ordering makes the set of points $p_{\pi_{1}, \pi_{2}}$ in $S$ into a directed system. Since $S$ is a compact space, this directed system has a point of accumulation $p$. Let $(x, y)_{p}$ denote the $(x, y)$ coordinate of $p$.

Then given a finite set of vector pairs $\left(x_{i}, y_{i}\right), i=1,2, \cdots, n$, and $\epsilon>0$, and a pair $\pi_{1}^{0}, \pi_{2}^{0}$ of partitions, we have 


$$
\left|\left(x_{i}, y_{i}\right)_{p}-\left(x_{i}, y_{i}\right)_{\pi_{1}, \pi_{2}}\right|<\epsilon \quad(i=1,2, \cdots, n)
$$

for some

$$
p_{\pi_{1}, \pi_{2}}>p_{\pi_{1}^{0}, \pi_{2}^{0}}
$$

Since $(x, y)_{\pi_{1}, \pi_{2}}$ is a scalar product for all $\pi_{1}, \pi_{2}$ it thus follows that $(x, y)_{p}$ is a scalar product, and since the norm $\|x\|_{\pi_{1}, \pi_{2}}$ is equivalent to the original norm with constants of equivalence independent of $\pi_{1}, \pi_{2}$, it follows that

$$
\|x\|_{p}=\sqrt{(x, x)_{p}}
$$

is also equivalent to the original norm.

Finally, fix a Borel set $\sigma$ and vectors $x_{2} y$. Let $\pi_{1}^{0}$ be the partition defined by $\sigma$ and its complement, and $\pi_{2}^{0}$ be arbitrary. Then, if

$$
p_{\pi_{1}, \pi_{2}}>p_{\pi_{1}^{0}, \pi_{2}^{0}}
$$

we have

$$
(E(\sigma) x, y)_{\pi_{1}, \pi_{2}}=(x, E(\sigma) y)_{\pi_{1}, \pi_{2}},
$$

since $\pi_{1}$ is a refinement of $\pi_{1}^{0}$, and so $\sigma$ is a finite union of sets involved in the partition $\pi_{1}$. Thus

$$
(E(\sigma) x, y)_{p}=(x, E(\sigma) y)_{p},
$$

and so the $E(\sigma)$ are self-adjoint with respect to the scalar product $(x, y)$, and similarly the $F(\eta)$ are self-adjoint with respect to this scalar product.

Since $\|x\|_{p}$ is equivalent to the given norm, it now follows that there exists a bi-continuous operator $A$ with $(x, y)_{p}=(A x, A y)$, and hence $A E(\sigma) A^{-1}$ and $A F(\eta) A^{-1}$ are all self-adjoint.

4. Proof of Theorem 2. By Theorem 8 of [1], an operator $T$ is spectral if and only if there exist two commuting operators $S$ and $N$ such that $N$ is quasinilpotent and $S$ admits a representation:

$$
S=\int \lambda E(d \lambda),
$$

where $E(d \lambda)$ denotes integration with respect to a certain spectral measure. 
Such an $S$ is called in [1] a "scalar type operator."

Now, by hypothesis, $T_{1}$ and $T_{2}$ are commuting spectral operators. We write

$$
T_{1}=S_{1}+N_{1}, T_{2}=S_{2}+N_{2},
$$

in accordance with the preceding. Then by Theorem 5 of [1] the operators $S_{1}, S_{2}, N_{1}, N_{2}$ all commute with one another. We thus have

$$
T_{1}+T_{2}=S_{1}+S_{2}+? \text { and } T_{1} T_{2}=S_{1} S_{2}+Q^{\prime}
$$

where $Q$ and $Q^{\prime}$ are quasi-nilpotent, $Q$ commutes with $S_{1}+S_{2}$, and $Q^{\prime}$ commutes with $S_{1} S_{2}$. By Theorem 8, quoted above, it is thus sufficient to show that $S_{1}+S_{2}$ and $S_{1} S_{2}$ are spectral operators of type 0 ; that is, of scalar type.

Let $E^{1}(\sigma)$ and $E^{2}(\sigma)$ be the spectral measures for $S_{1}$ and $S_{2}$, respectively. By Theorem 5 of [1] it follows, from the fact that $S_{1} S_{2}=S_{2} S_{1}$, that $E^{1}(\sigma)$ and $E^{2}(\sigma)$ commute with one another for all $\sigma$. liy our Theorem 1, then, there exists an operator $A$ such that the operators $A E^{1}(\sigma) A^{-1}$ and $A E^{2}(\sigma) A^{-1}$ are all self-adjoint. Hence

$$
J_{1}=A S_{1} A^{-1} \text { and } J_{2}=A S_{2} A^{-1}
$$

are normal operators. Also $J_{1} J_{2}=J_{2} J_{1}$, since $S_{1} S_{2}=S_{2} S_{1}$. It follows that $J_{1}+J_{2}$ and $J_{1} J_{2}$ are again normal operators, for they commute with their adjoints as we verify by direct computation, using the fact that $J_{1}$ and $J_{2}^{*}$ commute and $J_{2}$ and $J_{1}^{*}$ commute, since $J_{1}$ and $J_{2}$ commute.

Thus $A\left(S_{1}+S_{2}\right) A^{-1}$ and $A\left(S_{1} S_{2}\right) A^{-1}$ are normal operators and so of scalar type. But if $J$ is a scalar type operator and $A$ bi-continuous, then, as is easily seen, $A^{-1} J A$ is again a scalar type operator. Hence $S_{1}+S_{2}$ and $S_{1} S_{2}$ are scalar type operators, and all is proved.

\section{REFERENCES}

1. N. Dunford, Spectral operators, Pacific J. Math. 4 (1954), $321-354$.

2. E. R. Lorch, Bicontinuous linear transformations in certain vector spaces, Bull. Amer. Math. Soc. 45 (1939), 564-569.

3. G.W. Mackey, Commutative Banach algebras, multigraphed Harvard lecture notes, edited by A. Blair, 1952. 



\section{PACIFIC JOURNAL OF MATHEMATICS}

\section{EDITORS}

\author{
M.M. SCHIFFE R* \\ Stanford University \\ Stanford, California \\ E. HEWITT \\ University of Washington \\ Seattle 5, Washington
}

R.P. DILWORTH

California Institute of Technology Pasadena 4, California

E.F. BECKENBACH**

University of California

Los Angeles 24, California

\section{ASSOCIATE EDITORS}

$\begin{array}{llll}\text { H. BUSEMANN } & \text { P.R. HALMOS } & \text { BORGE JESSEN } & \text { J. J. STOKER } \\ \text { HERBERT FEDERER } & \text { HEINZ HOPF } & \text { PAUL LÉVY } & \text { E.G. STRAUS } \\ \text { MARSHALL HALL } & \text { R.D. JAMES } & \text { GEORGE PÓLYA } & \text { KÔSAKU YOSIDA }\end{array}$

\section{SPONSORS}

UNIVERSITY OF BRITISH COLUMBIA CALIFORNIA INSTITUTE OF TECHNOLOGY UNIVERSITY OF CALIFORNIA, BERKELEY UNIVERSITY OF CALIFORNIA, DAVIS UNIVERSITY OF CALIFORNIA, LOS ANGELES UNIVERSITY OF CALIFORNIA, SANTA BARBARA UNIVERSITY OF NEVADA OREGON STATE COLLEGE UNIVERSITY OF OREGON
UNIVERSITY OF SOUTHERN CALIFORNIA STANFORD RESEARCH INSTITUTE STANFORD UNIVERSITY WASHINGTON STATE COLLEGE UNIVERSITY OF WASHINGTON

AMERICAN MATHEMATICAL SOCIETY HUGHES AIRCRAFT COMPANY

Mathematical papers intended for publication in the Pacific Journal of Mathematics should be typewritten (double spaced), and the author should keep a complete copy. Manuscripts may be sent to any of the editors. Manuscripts intended for the outgoing editors should be sent to their successors. All other communications to the editors should be addressed to the managing editor, E.G. Straus, at the University of California Los Angeles 24, California.

50 reprints of each article are furnished free of charge; additional copies may be obtained at cost in multiples of 50 .

The Pacific Journal of Mathematics is published quarterly, in March, June, September, and December. The price per volume (4 numbers) is $\$ 12.00$; single issues, $\$ 3.50$; back numbers (Volumes $1,2,3$ ) are available at $\$ 2.50$ per copy. Special price to individual faculty members of supporting institutions and to individual members of the American Mathematical Society: $\$ 4.00$ per volume; single issues, $\$ 1.25$.

Subscriptions, orders for back numbers, and changes of address should be sent to the publishers, University of California Press, Berkeley 4, California.

Printed at Ann Arbor, Michigan. Entered as second class matter at the Post Office, Berkeley, California.

* To be succeeded in 1955, by H.L. Royden, Stanford University, Stanford, California.

** To be succeeded in 1955, by E.G. Straus, University of California, Los Angeles 24, Calif.

UNIVERSITY OF CALIFORNIA PRESS - BERKELEY AND LOS ANGELES 


\section{Pacific Journal of Mathematics \\ Vol. 4, No. $3 \quad$ July, 1954}

Nelson Dunford, Spectral operators ........................ 321

John Wermer, Commuting spectral measures on Hilbert space.......... 355

Shizuo Kakutani, An example concerning uniform boundedness of spectral measures...................................... 363

William George Bade, Unbounded spectral operators .............. 373

William George Bade, Weak and strong limits of spectral operators ...... 393

Jacob T. Schwartz, Perturbations of spectral operators, and applications. I. Bounded perturbations ........................... 415

Mischa Cotlar, On a theorem of Beurling and Kaplansky............... 459

George E. Forsythe, Asymptotic lower bounds for the frequencies of certain polygonal membranes ............................... 467 